\title{
La interface del cuidado de enfermería con las políticas de atención al anciano
}

\author{
A interface do cuidado de enfermagem com as políticas de atenção ao idoso \\ The interface of nursing care with the aged attention policies
}

\begin{abstract}
Juan José Tirado Darder', Zuila Maria de Figueiredo Carvalho"
' Universidad Cardenal Herrera, Colegio de Enfermería de Valencia, Núcleo de Investigación y Extensión en Enfermería (Miembro Pesquisador). Valência, Espanha.

"Universidade Federal do Ceará, Departamento de Enfermagem, Programa de Pós-Graduação em Enfermagem. Fortaleza-CE, Brasil.
\end{abstract}

\author{
Submissão: 23-11-2012 Aprovação: 24-11-2012
}

\section{RESUMEN}

Se objetivó hacer una explanación sobre la interface del cuidado de enfermería con las políticas de atención al anciano. Presenta-se el envejecimiento como una realidad mundial y una victoria de la sociedad moderna; la situación de las personas ancianas en España y las consecuencias sociales del envejecimiento en España; dependencia y necesidades de asistencia; situación de las personas ancianas en Brasil; comparación entre España y Brasil; niveles de dependencia; las soluciones que suministran las enfermeras y atención domiciliaria como base de un mejor futuro. Las consideraciones apuntadas son: La atención a la dependencia debe ser abordada de inmediato, considerar los fracasos en otros países, para no caer en los mismos errores, instar a la población mayor al mantenimiento de su independencia con promoción de la salud.

Palabras clave: Envejecimiento; Anciano; Enfermería de Atención Primaria.

\section{RESUMO}

Objetivou-se fazer uma explanação sobre a interface do cuidado de enfermagem com as políticas de atendimento ao idoso. Apresenta-se o envelhecimento como uma realidade mundial e uma vitória da sociedade moderna; a situação das pessoas anciãs e as consequências sociais do envelhecimento na Espanha; a dependência e necessidades de assistência; a situação das pessoas anciãs em Brasil; comparação entre a Espanha e o Brasil; níveis de dependência; as soluções que as enfermeiras fornecem e o atendimento domiciliário como base de um melhor futuro. As considerações apontadas são: o atendimento à dependência deve ser abordada de imediato, considerar os fracassos em outros países, para não cair nos mesmos erros, instar a população de idosos à manutenção de sua independência com promoção da saúde.

Descritores: Envelhecimento; Idoso; Enfermagem de Atenção Primária.

\section{ABSTRACT}

The objective was to make an explanation on the interface of nursing care with the elder care policies. It is presented the aging phenomenon as a global reality and a victory of modern society; the situation of elderly people and the social consequences of aging in Spain; the dependence and assistance needs; the situation of elderly people in Brazil; comparison between Spain and Brazil; dependency levels; and the solutions that nurses provide and home care as a basis for a better future. The considerations given are: meeting the dependency must be addressed immediately, considering the failures in other countries, to avoid the same mistakes, and to urge the elder population to maintain their independence with health promotion.

Key words: Aging; Aged; Primary Care Nursing.

Conferência de abertura da $9^{a}$ Jornada Brasileira de Enfermagem Geriátrica e Gerontológica, promovida pela ABEn Nacional e realizada pela ABEn Seção Ceará, ocorrida, em setembro de 2012, em Fortaleza-CE, Brasil. 


\section{INTRODUCCIÓN}

El envejecimiento de la población difiere del envejecimiento de las personas, una vida larga es un signo de buena salud. El envejecimiento de la población mundial, tanto en los países desarrollados como en los países en desarrollo, resulta un indicador de la mejora de la salud mundial. En la actualidad, la población mundial de 60 años o más es de 650 millones, y se calcula que en 2050 alcanzará los 2000 millones; de estos, aproximadamente un $80 \%$ de las personas mayores vivirán en países poco desarrollados. El envejecimiento de la población corre en paralelo a la urbanización rápida: en 2007 más de la mitad de la población mundial vivía en ciudades, y se calcula que en 2030 la cifra será superior al $60 \%$.

Sin embargo, esta tendencia positiva se acompaña de retos sanitarios especiales para el siglo XXI, por lo que resulta imprescindible preparar a los dispensadores de atención sanitaria, y a la sociedad misma, para que contemplen las necesidades de la población mayor con una formación específica dirigida a los profesionales sanitarios que los atienden. Dicha formación debe centrarse en la promoción de la salud, la prevención y tratamiento de las enfermedades crónicas asociadas a la edad, la formulación de políticas sostenibles en materia de atención a largo plazo, la atención paliativa y el desarrollo de servicios y entornos adaptados a las necesidades de las personas mayores.

Con estas premisas podemos afirmar que:

El envejecimiento de la población es un triunfo de la sociedad moderna. Reflejo claro de la mejora de la salud mundial pero que, a su vez, plantea retos especiales para el siglo XXI. La esperanza de vida en países como Japón y Francia estaba por encima de los 80 años ya en 2005, produciéndose también un aumento en los países en desarrollo. Por ejemplo, un niño que nazca hoy en Chile, Costa Rica, Jamaica, Líbano, Sri Lanka o Tailandia, puede aspirar a una longevidad superior a los 70 años.

En 2050, cerca del $80 \%$ de las muertes corresponderán a personas mayores de más de 60 años. Los gastos sanitarios aumentan con la edad, concentrándose en el último año de vida. Cuanto más tarde muera una persona, menores serán los costes aglutinados en ese periodo. El aplazamiento de la edad de la muerte gracias a un envejecimiento saludable y a unas políticas adecuadas para el final de la vida, podría proporcionar grandes ahorros en materia de atención sanitaria.

La inversión en salud a lo largo de la vida produce dividendos a cualquier sociedad. Las personas mayores también representan un recurso para sus familias, comunidades y economías. Muy pocas veces es demasiado tarde para cambiar comportamientos de riesgo y promover la salud

Para promover la salud, prevenir la enfermedad y tratar las enfermedades crónicas, es fundamental una atención primaria eficaz a las personas mayores. En general, la formación de los profesionales sanitarios tiene un pequeño o nulo contenido en materia de atención a las personas mayores. Sin embargo, el tiempo que pasan atendiendo a este sector de la población va en aumento. La OMS sostiene que todos los prestadores de atención sanitaria deben poseer formación geriátrica y gerontológica, independientemente de cuál sea su profesión.

El maltrato a los ancianos está aumentando a medida que la población envejece y cambia la dinámica social. La OMS calcula que entre un $4 \%$ y un $6 \%$ de las personas mayores han sufrido alguna forma de maltrato: físico, psicológico, emocional, financiero o debido a negligencias. El maltrato de los ancianos constituye una violación de los derechos humanos ${ }^{(1)}$. La tendencia actual hacia el envejecimiento de la sociedad es clara, suscitando una gran cantidad de informes en todo el mundo. Esta afirmación está lo suficientemente respaldada por innumerables estudios, tanto los realizados por Naciones Unidas como por la Comisión Europea o un buen número de instituciones y universidades de reconocido prestigio, a lo largo de los últimos años.

Como queda reflejado, resulta evidente que el envejecimiento de la sociedad es un hecho. La Organización de las Naciones Unidas recoge en su informe "Envejecimiento de la Población" la observación de que se trata de un fenómeno sin precedentes y generalizado, que afecta a prácticamente todos los países del mundo. Las causas serían la disminución de la natalidad, por un lado, y el aumento de la esperanza de vida por el otro. Además, indica que el proceso se manifiesta de manera continua desde el año 1950, siendo el grupo de edad que presenta el crecimiento mas rápido del mundo el de los más mayores, es decir, los que tienen más de 80 años.

Indudablemente, y por necesidad, hemos de afrontar este desafío bajo todas las perspectivas posibles si queremos mantener el estado del bienestar. Estos planteamientos deben dejar de lado el personalismo de los distintos roles profesionales y académicos, centrándose en la solución de los problemas a partir de equipos multidisciplinares, capaces de mejorar y dar continuidad al sistema, con propuestas cercanas al usuario. De este modo, es posible conseguir su implicación a la hora de generalizar un envejecimiento activo y saludable.

En el año 2010, el IMSERSO presentó un documento llamado "El Libro Blanco del Envejecimiento Activo"(2), en el que se proponen una serie de líneas de actuación, destacando la importancia de la tecnología en estos tres ámbitos: 1.Educación a lo largo de la vida, 2.Diversidad y participación, y 3. Formas de vida y convivencia.

Resulta extremadamente complicado que nuestros mayores se adapten a las nuevas tecnologías, de ahí la importancia de comenzar con su aplicación en edades más tempranas, en las que sus capacidades aún les permiten asimilar la gran ventaja que representa su utilización. De esta manera, se constata el valor que, a su vez, supone para el sistema, dado que permite mejorar los procesos y el control de los mismos, lo que se traduce en una optimización de los recursos.

En este contexto, enfermería, de una vez por todas, debe demostrar su valía e implicación en todo lo que su responsabilidad exige, sin ningún tipo de complejos y aportando su ciencia en los cuidados y la promoción de la salud, convirtiéndose, en definitiva, en el motor impulsor de la racionalización del sistema en todos los países donde haya alcanzado su madurez, tanto académica como profesional. 


\section{EL ENVEJECIMIENTO, UNA REALIDAD MUNDIAL}

La Primera Asamblea Mundial sobre el Envejecimiento se realizó en 1982, demostrando la preocupación que todos los países tienen con una realidad tan inminente ${ }^{(3)}$. Veinte años después, en 2002, la División de Población del Departamento de Asuntos Económicos y Sociales de las Naciones Unidas manifestó, en un estudio, que la cuestión del envejecimiento era un fenómeno a escala planetaria. Dicho estudio ha sido revisado en varias ocasiones, la última en el año 2009, lo que le concede una importantísima relevancia en cualquier aproximación a esta problemática.

Durante estas revisiones se ha puesto énfasis en destacar algunos aspectos que, por su importancia, pasamos a comentar ${ }^{(4)}$ :

$\checkmark \quad$ El fenómeno del envejecimiento actual no tiene precedentes en la historia de la humanidad. La representación gráfica de esta realidad, muestra el cambio de lo que se
Ilamaba pirámide poblacional a lo que puede ser considerado un "hongo" poblacional, ya que el número de personas mayores superará, en el año 2045, al de los niños.

$\checkmark \quad$ Esta estimación afectará, prácticamente, a todos los países del planeta, lo que puede llevar a la equidad y solidaridad inter e intrageneracional, de acuerdo a sus bases educacionales.

$\checkmark \quad$ Este cambio demográfico tendrá consecuencias en todos los aspectos; sociales, económicos y políticos. En los países democráticos, el voto se verá afectado por una población mayoritariamente de edad avanzada, que defenderá un estatus actualmente impensable, influyendo en todos los órdenes; sobre la familia y, también, en las relaciones sociales.

\section{Situación en España}

España es, actualmente, uno de los países con un ritmo de envejecimiento más rápido.

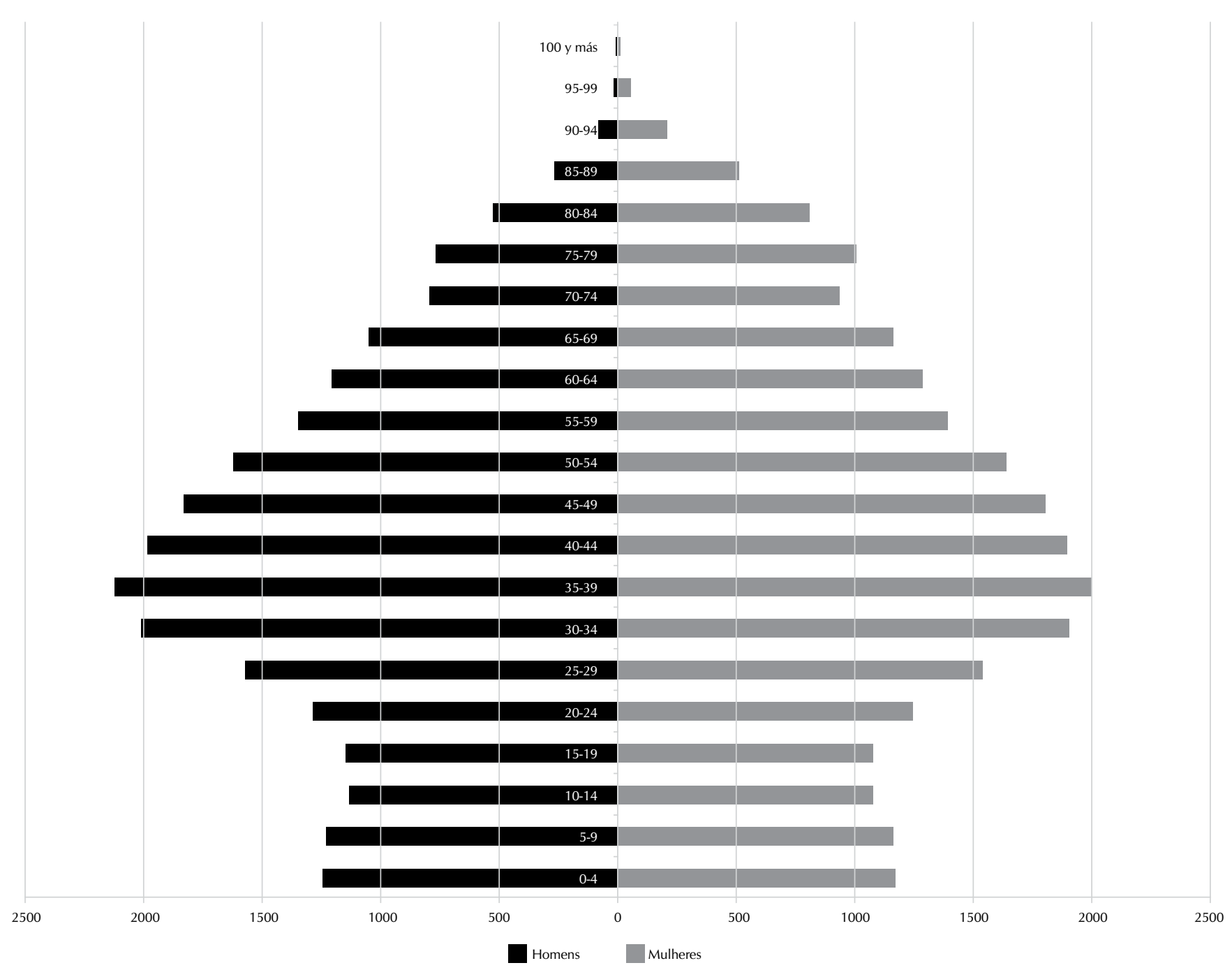

Figura 1 - Pirámide población española 2012. Fuente INE 
Como se aprecia en la figura 1, en España crece la proporción de personas mayores de 64 años. Los datos recogidos han demostrado que entre 1975 y 2010 ha pasado del $10 \%$ al $17 \%$, y esta progresión seguirá aumentando en las próximas décadas.

La pirámide de 2012 refleja el acusado descenso de la natalidad, prolongado durante 20 años, destacándose ya las edades adultas centrales (generaciones del baby boom). Unas generaciones que, para el año 2050, serán mayoritarias en esta pirámide, que pasa a parecerse cada vez más a un hongo. Las consecuencias sociales del envejecimiento en España son: feminización - la diferencia de mortalidad entre hombres y mujeres desequilibra la relación numérica entre sexos; sobreenvejecimiento - crece el número de personas que alcanza edades muy avanzadas, y mayor dependencia - dado que los problemas de salud guardan una relación directa con la edad, la morbilidad aumenta.

España ha demostrado ser pionera en materia de envejecimiento activo. Participamos dinámicamente con la ONU en la organización de la II Asamblea Mundial sobre Envejecimiento, celebrada en Madrid en el año 2002, así como en la organización de la Conferencia Ministerial de la Región Económica para Europa de las Naciones Unidas (CEPE), sobre envejecimiento, celebrada en León en el año 2007.

De esta última conferencia se deriva la Declaración Ministerial de León, que recoge una serie de compromisos de los gobiernos de los países miembros de la CEPE para avanzar en materia de envejecimiento. Entre otras actuaciones, esta Declaración Ministerial promueve las destinadas a la prevención y atención de la salud, a fomentar el envejecimiento activo, la vida independiente, la participación social y la educación a lo largo de la vida, cuestiones éstas que no han sido respetadas, tal como se planteó con la creación de la llamada Ley de la Dependencia.

La Ley de Promoción de la Autonomía Personal y Atención a las Personas en Situación de Dependencia y a las Familias de España, más conocida como "ley de dependencia"(5), es una ley española que sienta las bases para construir el futuro Sistema Nacional de Atención a la Dependencia, que financiará los servicios que necesitan las personas dependientes, bien por sufrir una enfermedad o accidente invalidante, o bien al llegar a la vejez, considerando la dependencia personal como la incapacidad funcional para el desarrollo de actividades de la vida diaria y el consiguiente requerimiento de ayuda para su realización.

La Ley fue presentada el 5 de marzo de 2006 por el entonces presidente del Gobierno, José Luis Rodríguez Zapatero, en un acto público. El 20 de abril de 2006, fue aprobada por el Consejo de Ministros, y el 30 de noviembre de 2006 se aprobó de forma definitiva en el Pleno del Congreso de los Diputados con una amplia mayoría, pero, curiosamente, al contrario de lo ocurrido en 1986 con la Ley General de Sanidad, que convirtió a España en la mejor sanidad del mundo, esta ley de 2006 no se aprobó acompañada de un presupuesto que la hiciera posible. Tanto es así que, en este año 2012, la propia ministra de Sanidad, Ana Mato, afirmó el 12 de abril que la ley "no ha contribuido a crear empleo, sino todo lo contrario".
De hecho se han perdido 8.305 puestos en servicios sociales. Además, la ley ha provocado la creación de 17 sistemas distintos de dependencia en las comunidades autónomas, produciéndose un desfase entre las previsiones y el número real de dependientes. Por todo ello, es necesaria una profunda reforma del Sistema de Autonomía Personal y Atención a la Dependencia, para hacerla viable en todo el territorio nacional.

La realidad es ésta: la ley de Dependencia permitía, como excepción, las prestaciones económicas al dependiente. Eso favorecía que la familia cuidara al anciano o dependiente a cambio de las prestaciones económicas de Dependencia.

Esta situación contemplaba el cuidado del dependiente por personal no preparado. $Y$ lo que al final ha sucedido es que una situación excepcional ha terminado por ser la norma general que ha imperado en la ley de Dependencia. El fracaso de esta ley ha sido desencadenado por la crisis económica; las familias, asfixiadas en una situación de crisis, han intentado recaudar medios de dónde han podido y, visto que si cuidaban ellos mismos a la persona dependiente podrían percibir las prestaciones de la ley de Dependencia, renunciaban así a los servicios asistenciales, que son los que realmente están preparados para ofrecer el cuidado al dependiente. Por ello, se debe "potenciar la utilización de cuidadores familiares cuando así lo requieran las familias que quieren tener a la persona dependiente en su casa, pero siempre que esta persona pueda estar bien atendida en su domicilio".

Esta realidad, asociada a otras actuaciones, como la de centralizar la atención al ingreso en Residencias, la mayor parte de ellas concertadas o privadas, consiguió que se generara un boom de este tipo de establecimientos, lo que multiplicó su crecimiento como negocio, con las particularidades que conlleva.

En la situación actual, y con la profunda crisis económica que nos azota, son muchos los establecimientos residenciales que han cerrado o que no son capaces de mantenerse, generando un cambio importante en la estructura asistencial al mayor. Ello, a su vez, está condicionando y focalizando la atención a la Dependencia en lo que realmente siempre ha aconsejado la Ley, es decir, en "la atención domiciliaria y la promoción de la autonomía personal", como herramienta indiscutible para el mantenimiento del individuo y, por ende, del Estado del Bienestar.

Es ahora cuando, desde el punto de vista de la Enfermería, se pueden ofertar las soluciones que nuestros conocimientos avalan, ofreciendo una racionalización del sistema y un abaratamiento del mismo, como luego podremos ver.

Número de personas dependientes en España en el año 2012

En abril de 2012, y según los últimos datos disponibles del Instituto Nacional de Estadística (INE), España contaba con 7.358.726 personas mayores (de 65 o más años), de las que 2.227.086, el 30,3\%, sufren alguna discapacidad. De ellas, 1.400.293, el 19\%, están en situación de dependencia (necesitan de la asistencia de otra persona). En este último grupo de dependientes, el $71 \%$ son mujeres, el $62,1 \%$ analfabetos o sin estudios, y el $54,1 \%$ tiene 80 o más años de edad ${ }^{(6)}$ 


\section{Dependencia y necesidades de asistencia}

La discapacidad está asociada a restricciones que afectan todos los aspectos de la vida, pero no todas las personas con discapacidad requieren la ayuda de una tercera persona ${ }^{(7)}$.

De forma generalizada, podemos afirmar que la proporción de mayores discapacitados con necesidades no cubiertas varía entre el $2 \%$ y el $35 \%$, según el tipo de necesidades consideradas $^{(8)}$. Si bien es cierto que en los países desarrollados, la tecnología ha evitado en gran parte que estas cifras se disparen, ya que la utilización de ayudas técnicas es mayor, disminuyendo la necesidad de ayuda por parte de terceras personas. Todo esto nos indica que las ayudas técnicas serán también cada vez más aceptadas por la población mayor europea, y que las demandas de ayuda de una tercera persona tenderán a disminuir.

En las últimas dos décadas del siglo XX, en la mayoría de los países de altos ingresos se ha observado una disminución de la prevalencia de la discapacidad, acompañada de un aumento en la prevalencia de los trastornos crónicos. Las mejoras de las condiciones de vida y de los cuidados sanitarios podrían explicar estas observaciones. Hay grandes desigualdades socioeconómicas y de género en el origen de la discapacidad y en las necesidades no cubiertas de las personas mayores discapacitadas.

\section{Situación en Brasil}

Aun no siendo el mismo panorama que se ha presentado en España, Brasil atraviesa actualmente un inédito proceso, en el que se estima que para 2020 la población más vieja predominará sobre la de menos edad; "Brasil se encuentra en medio de una profunda transformación socioeconómica impulsada por sus cambios demográficos ${ }^{(9)}$, lo que obligará al gobierno a impulsar cambios drásticos en sus políticas sociales, cuestión que, sin duda, le permite desechar las políticas que otros países han probado con efectos negativos.

El fenómeno es llamado "bono demográfico", y se produce cuando "la fuerza laboral es mucho menor a la población dependiente".

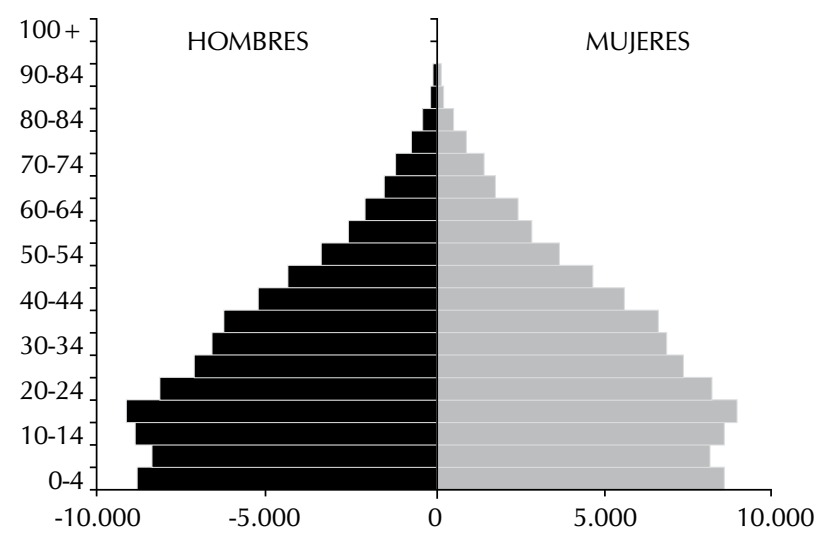

Figura 2 - Pirámide población brasileña 2000. Fuente: Naciones Unidas
“La población anciana crecerá más del triple en las próximas décadas; de menos de 20 millones en 2010 pasará, aproximadamente, a 65 millones, en 2050".

Como se aprecia en la figura 2, la población brasileña en el año 2000 mantenía una estructura gráfica piramidal, que reflejaba una ligera hegemonía de la población entre los 10 y los 20 años.

Las previsiones presentadas en la figura 3, muestran que existe una tendencia igualar los tramos de edad hasta los 45 años, con un claro aumento de la población de mayor de 60 años.

Ya en la proyección del año 2050, donde vemos como el incremento de la población mayor representará un impacto importante para la economía y la situación social del país. Lo que ya no es una previsión, es el hecho de que la población de Brasil alcanzó en 2010 los 190,7 millones de personas, una cifra que se estima aumentará a 215,3 millones en los próximos 40 años, según datos del Instituto Brasileño de Geografía y Estadística (IBGE).

En su informe, el Banco Mundial señala que la tasa de mortalidad en Brasil disminuyó, principalmente, entre los más jóvenes, pero también lo hizo la de fecundidad. Así, "una mujer brasileña promedio tenía más de seis hijos a comienzos de la década de los 60 y actualmente sólo tiene más de dos". En consecuencia, se estima que, para mediados de 2020, la tasa de crecimiento del grupo de personas entre 15 y 59 años será negativa y el "aumento poblacional se verá impulsado por el aumento del número de ancianos". Este cambio demográfico se traducirá en un crecimiento económico, ya que al disminuir la tasa de fecundidad, más mujeres se integrarán al mercado de trabajo o invertirán su dinero en la educación. Por todo ello, y paralelamente a esta previsión, Brasil debe impulsar cambios drásticos en sus políticas sociales, pues este "corto" fenómeno tendrá definitivamente un impacto socioeconómico en el país.

“Brasil se embarcó en un proceso de desarrollo que lo está llevando a alcanzar índices sociales y demográficos de primer mundo, pero con sistemas e instituciones heredadas de otro contexto". El cambio debe comenzar en las políticas fiscales,

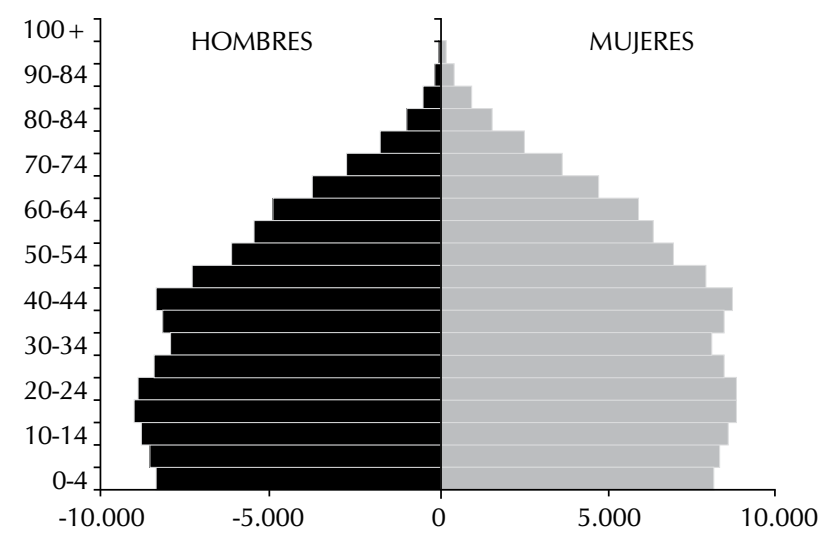

Figura 3 - Pirámide población brasileña 2025. Fuente: Naciones Unidas 
pues un aumento de la población vieja puede terminar barriendo el crecimiento proyectado, por ejemplo, con la propagación de las "jubilaciones precoces".

El BM calcula que para 2050, el pago de pensiones representará una cifra equivalente al 22,4\% del PIB brasileño.

\section{Comparación entre España y Brasil}

Aunque las comparaciones son difíciles de realizar entre dos países tan diferentes, vamos a intentar hacer una comparación que nos permita observar el desarrollo que ambos han tenido en políticas de salud y el impacto que sobre las economías ha producido.

Observamos, en la figura superior 4, la diferencia existente entre la población de ambos países, ya que la brasileña es tres veces superior a la española (eso sí, somos campeones del mundo en fútbol).

Los mayores de 60 años, en la actualidad, representan un total del $10 \%$ de la población brasileña mientras que en España, hablamos de un $22 \%$. A la hora de comparar las tasas de natalidad, la población brasileña alcanza un 15,5 por cada 1000, mientras la española representa el 10,9 de cada 1000, teniendo en cuenta que durante estos últimos diez años ha aumentado gracias a la población inmigrante, algo que, seguramente, sufrirá un descenso a causa de la crisis y la vuelta de los inmigrantes a sus países de origen.

Por último, y debido al envejecimiento de la población principalmente, nos encontramos con que el índice en la tasa de mortalidad, en España, supera en dos puntos al de Brasil. La evolución del gasto sobre el PIB queda reflejada en la siguiente imagen, gráfico 1, que recoge los datos generados por estadísticas sanitarias mundiales en $2012^{(10)}$.
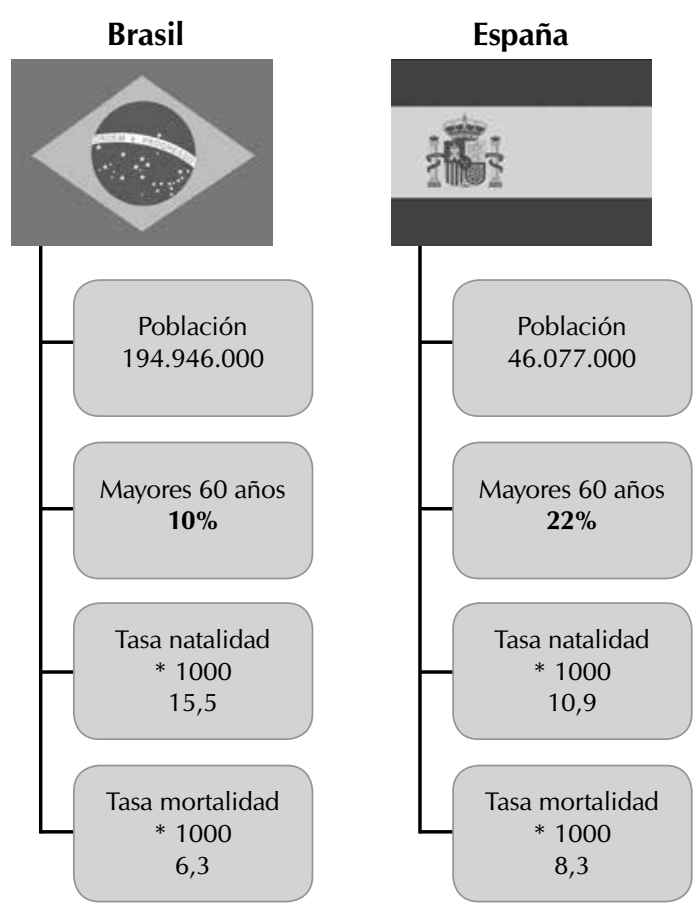

Figura 4 - Comparación Brasil España. Fuente: elaboración propia.
Se aprecia que en el año 2000 el gasto en salud de ambos países suponía un 7,20\% del PIB, mientras que en el año 2009, España superaba en casi un punto a Brasil en gasto sanitario.

Las diferencias entre "personal de bata blanca" (desconozco si en Brasil se hace referencia solamente a las enfermeras o están incluidas las auxiliares, pero en España se presentan únicamente los datos de las enfermeras).

Como se puede ver, la diferencia entre colectivos de bata blanca entre Brasil y España es bastante grande. En cuanto a las auxiliares de Enfermería, en España no pueden realizarse estimaciones concretas al no contar con fuentes suficientes para realizar la valoración, ya que no existen datos de colegiación por carecer de colegio profesional y, únicamente, disponemos de las cifras de la EESCRI, que no incluyen los centros sanitarios en régimen ambulatorio ${ }^{(11)}$. Aun así, se baraja que el número de auxiliares es aproximadamente tres veces superior al de enfermeras.

\section{Niveles de dependencia}

La tasa de dependencia es el índice demográfico que expresa, en forma de tasa y en porcentaje, la relación existente entre la población dependiente y la población productiva, de la que aquélla depende. En las estadísticas internacionales, se suele simplificar la definición de ambas poblaciones en términos de edad, obviando el hecho de que una parte importante de la población en edad laboral no es población activa (es decir, no está incorporada al mercado de trabajo por una u otra razón ${ }^{(12)}$. A medida que la tasa se incrementa, aumenta la carga que supone para la parte productiva de la población el mantener a la parte económicamente dependiente; por un lado, los niños, y por otro, los ancianos. Las previsiones presupuestarias en educación, sanidad, pensiones y otros gastos sociales, deben ajustarse como consecuencia de ello, además de cambiar su composición.

Con respecto a la población anciana, sus demandas aumentan progresivamente, debido a que un colectivo cada vez más amplio está exigiendo servicios de salud. Esto presiona al sistema de seguridad social, que necesitará cada vez más la ayuda de instituciones de apoyo. La creciente longevidad de los adultos mayores también debe analizarse cuidadosamente, porque se perfila un gran desafío: la posibilidad de que la esperanza de vida experimente importantes prolongaciones sin que, a su vez, mejore la calidad de vida o incluso se deteriore por conformismo de los propios mayores, al considerar que es normal su estado. Podríamos decir que son ellos mismos los que padecen de "ancianismo", entendiendo que es la propia sociedad la responsable de buscar solución a sus deficiencias.

La discapacidad tiene un proceso, que define a los mayores en su aceptación y conformismo, incluso en su cultura de autosuficiencia al margen de su funcionalidad, haciendo que cada individuo adopte una conducta que marcará su umbral de discapacidad.

Finalmente, los cambios en el perfil etario de la población, exigen una reorientación de las políticas públicas, a fin de adaptar la distribución de los recursos a la nueva realidad de las demandas sociales. 
Visto lo visto, es preciso que las enfermeras se involucren en el desarrollo de soluciones a esta problemática de una manera directa y dirigiendo los cambios, ya que el área a desarrollar es la de la promoción de la salud y los cuidados, responsabilidad directa que, tanto los dirigentes políticos, como el resto de roles profesionales, deben aceptar.

\section{Soluciones que aportan las enfermeras}

Resulta escandaloso que, viendo el fracaso generalizado acontecido en los países más desarrollados y a la vez más viejos, se siga medicalizando la atención, destinando mayor cantidad de recursos al área paliativa que a la educativa y de promoción de la salud poblacional. Por todo ello, es fundamental que las enfermeras, avaladas por las cifras, tanto cuantitativas como cualitativas, de sus actuaciones y estudios, se conviertan en el motor del cambio necesario para el mantenimiento de las estructuras de apoyo a las personas mayores y dependientes.

La atención al mayor es algo a lo que debemos prepararnos desde la juventud. Por lo tanto, es preciso resaltar la importancia que tiene la enfermera escolar, una figura que, bajo su doble vertiente, asistencial y educativa, genera una base sólida en la formación que permite afrontar una vida saludable en prevención y promoción de la salud.

\section{La atención domiciliaria como base de un futuro mejor}

En el modelo de Servicio de Atención a Domicilio propuesto por la Fundación Edad y Vida, organización Española que analiza el desarrollo de este tipo de servicios, se llega a la conclusión de que éste debe tratarse de un modelo de atención integral sociosanitaria a las personas mayores en situación de dependencia. Se deben cubrir, por lo tanto, las necesidades de carácter social y sanitario, incluyendo servicios que vayan desde la promoción, la prevención, la atención y la rehabilitación, con el objetivo último de fomentar la permanencia de la persona en su entorno, manteniendo su red natural de apoyo. El modelo tiene como eje la libertad de elección del usuario en cuanto al proveedor del servicio, el cual debe estar acreditado, certificando su calidad. Esto último, así como la regulación de los profesionales prestadores, busca la profesionalización del sector. Asimismo, el modelo fomenta la corresponsabilidad de los usuarios en la sostenibilidad del sistema, mediante su participación en la financiación de los servicios. Finalmente, afirma que las Administraciones Públicas juegan un papel clave como garantes de la provisión de asistencia.

A nivel de regulación, el modelo considera necesario el establecimiento de un marco básico estatal que defina las características básicas y comunes.
Pasos a seguir en la valoración domiciliaria, producto creado y diseñado a partir del análisis y reflexión sobre los resultados obtenidos, se muestran los pasos a seguir de manera secuencial en una valoración, ofreciendo una atención holística que involucra a todos y cada uno de los miembros del equipo sociosanitario, así como al usuario, a los familiares e incluso a los cuidadores informales, dirigidos y coordinados, en cuanto a cuidados, por la figura de la enfermera.

En primer lugar, se define la realización de una valoración Geriátrica y Gerontológica holística, que aglutine toda la información necesaria obtenida en la recogida de datos sobre el entorno, las ayudas informales, los recursos, etc.

De acuerdo con la valoración, se procederá a realizar el PIA-PAE, que indica de forma protocolizada los cuidados que las personas deben recibir, o realizar, para mantener su autonomía personal o bien, para la recuperación de sus funcionalidades en la realización de las ABVD. Todo ello, mediante una serie de instrucciones y consejos que faciliten la consecución de los objetivos. Dichas instrucciones han sido previstas por la enfermera en su valoración, implicando en la ejecución al propio usuario y a los cuidadores, tanto formales como informales.

Esa misma valoración, que en nuestra propuesta será automática y, por lo tanto, protocolizada, permite a la enfermera realizar en base a los resultados obtenidos y su criterio sanitario, la derivación en materia de salud a otros profesionales, que, tanto a nivel preventivo como paliativo, deben actuar sobre la necesidad o el problema detectado. Los profesionales enumerados, sin ser exhaustivos, en la izquierda del cuadro, pueden ir aumentando según las necesidades que revele la valoración.

La siguiente etapa pasaría por proporcionar información y formación, tanto a usuarios como a cuidadores, e incluso a las asociaciones vinculadas a los mismos, en la que, mediante los

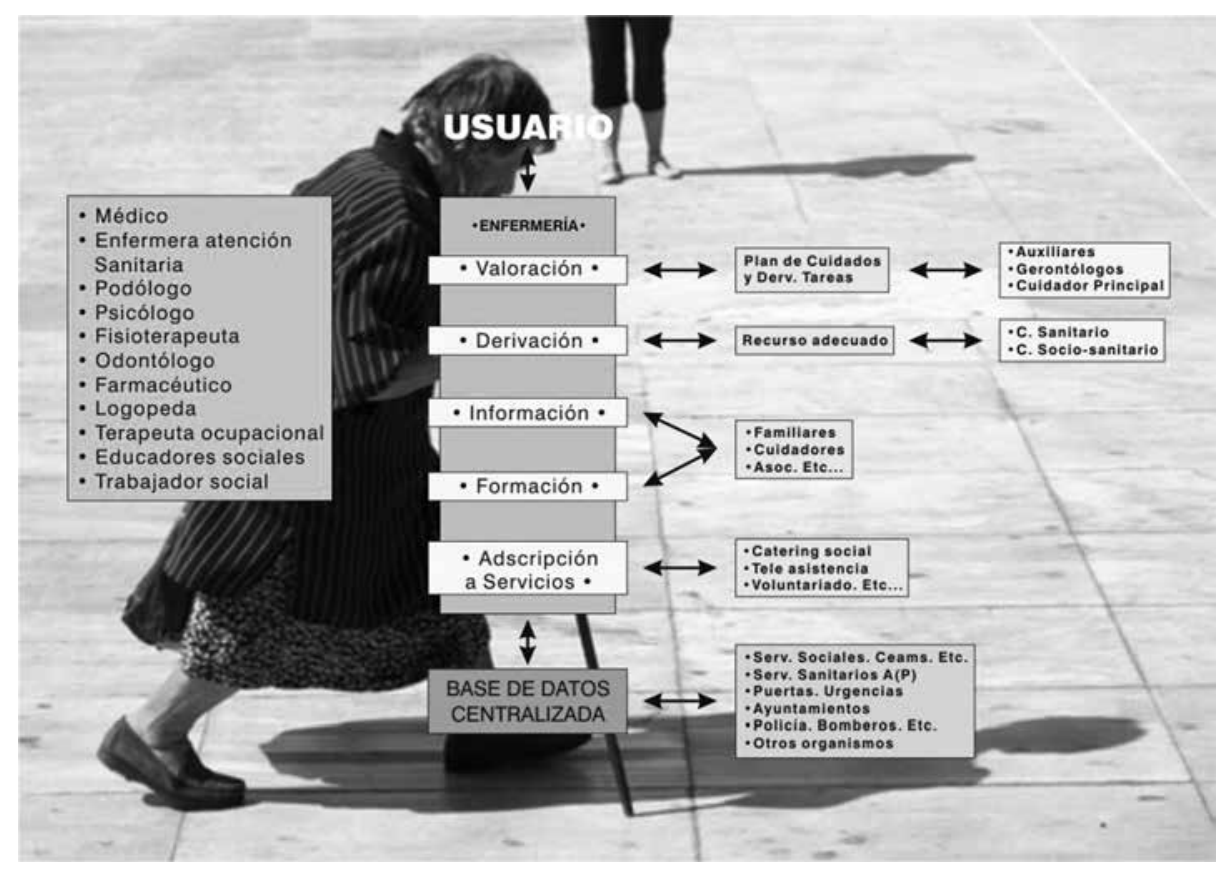

Figura 5 - Pasos a seguir en la valoración domiciliaria (Tirado-Darder, 2012). 
programas adecuados, se fomente la calidad de los cuidados y se despejen, por parte de todo el equipo multidisciplinar, todas aquellas dudas que puedan surgir y sea necesario disipar para mantener en perfecto estado de salud y comodidad a los dependientes, incluyendo la necesaria adecuación de los domicilios en los que viven.

En una última fase, se procederá a la adscripción a los servicios existentes en la comunidad que puedan beneficiar al usuario en su domicilio y le permitan mantenerse durante más tiempo en él. Entre ellos se encuentran desde la teleasistencia hasta el catering social, pasando por los servicios de atención en cuestiones básicas como la limpieza, por ejemplo.

Toda la información generada pasaría a formar parte de una base de datos centralizada que funcionaría como una ventanilla única. Una ventanilla que consideramos necesaria y en la deberían confluir todos aquellos organismos, profesionales y personas capaces de detectar la necesidad de que un dependiente sea atendido en su domicilio. Que evite, también, el que se produzcan situaciones similares a las que la prensa nos tiene acostumbrados; ancianos con Síndrome de Diógenes, abandonos, accidentes, muerte de personas que, al no solicitar ayuda, son desconocidos para las instituciones sociosanitarias, etc. Con ello, se conseguiría la trasformación de la demanda, que pasaría de ser generada por el usuario a ser generada por el profesional.

Para la realización del proceso de valoración y la realización de los planes de cuidados, es necesario recurrir a las TIC, y, desde enfermería, diseñar herramientas como las que vamos a presentar para que se agilice de forma evidente dicha tarea. Con ello se permite generar automáticamente un Plan de Atención estandarizado, con la posibilidad de individualizarlo para mantener, tal y como se indica en la ley de la Dependencia, la mayor autonomía posible, facilitando tanto el mantenimiento como la conservación de la misma, además de su recuperación.

Estas acciones deben elegidas por la enfermera, pudiendo ser llevadas a cabo tanto por ella misma como por el usuario, los familiares o cualquier otro cuidador. Acciones que se deben comenzar a realizar y controlar por cualquiera de los sujetos involucrados en el cuidado, y de las que deben informar a posteriori, en las siguientes visitas y valoraciones del profesional de enfermería de referencia.

De ahí la importancia de que sea el profesional de enfermería, individualmente y en colaboración, el que asuma las competencias, siendo capaz de contestar el "quién", "qué", "dónde", "cuándo" y "por qué" de las mediciones en los cuidados de asistencia a largo plazo, contando, a su vez, con los mejores instrumentos disponibles ${ }^{13)}$. Todo ello en base a que la provisión de los cuidados a nivel nacional mantenga una tendencia más sanitaria que social, Ministerio de Sanidad y Consumo $^{(14)}$ lo que demuestra, sin lugar a dudas, que la dependencia, hoy por hoy, tiene un marcado matiz sanitario, destacando el rol de la enfermera especializada en los cuidados sociosanitarios, siendo un profesional autónomo que coordina una atención compleja dirigida a personas en situación de dependencia, mayoritariamente compuesta por los ancianos y sus familias ${ }^{(15)}$.
La realización de este trabajo, en el que los profesionales de enfermería asumen el nivel máximo de responsabilidad, mejora la calidad de vida del usuario, lo que supone un impacto directo en la salud de las cuidadores/as, siendo ésta mejor, a su vez, en aquellos casos que cuentan con un buen apoyo familiar y social. El mantenimiento de un buen estado de salud y un buen nivel de actividad física, junto con sentimientos positivos, mejora las posibilidades de un afrontamiento positivo de la situación por parte de los cuidadores. Ello, a su vez, facilita la disponibilidad inmediata, a través del proceso de valoración informatizado, de un PIA-PAE adecuado y rápido.

Esta es una situación clave para el cuidador, ya que al sentirse respaldado por el sistema y tener la certeza de que existe una clara intervención, hace que las actuaciones y su correspondiente relación con la situación familiar, sea un importante factor predictor de la salud mental de los cuidadores de pacientes dependientes. Una situación que ha tenido y tendrá un impacto positivo en los dependientes atendidos.

Las necesidades de intervención deben completar las diferentes tipologías de atención, que, en nuestro caso, serían de aplicación al dependiente. Por todo ello, han sido diseñados una serie de mapas mentales que, incluidos en la herramienta informática, facilitan al profesional, y especialmente a los profesionales noveles, una guía de actuación que proporciona seguridad y exhaustividad en la cumplimentación de la valoración en general y del Índice de Barthel en particular. No basta con rellenar los ítems o preguntas que componen la valoración de forma mecánica, es también necesario tener los conocimientos sanitarios para que las respuestas indiquen claramente el nivel de dependencia del individuo, sin olvidar ninguno de los aspectos relevantes para la realización posterior del plan de cuidados. De ello depende el mantenimiento del máximo nivel de independencia del usuario, durante el mayor tiempo posible.

Esta estructura puede ser modificada, pero en el momento actual, consideramos que servirá de guía a todos los profesionales sanitarios que realicen valoraciones mediante la utilización de esta aplicación. Guías que pueden ser utilizadas junto con otras herramientas enfermeras, tales como los diagnósticos de enfermería (NANDA), las intervenciones enfermeras NIC y NOC y todos los indicadores precisos para llevar a cabo la valoración y realización holística de los planes de cuidados para el mantenimiento de la independencia de los individu${ } \mathrm{s}^{(16,17)}$. La terminología empleada en las guías utiliza descripciones muy básicas de los conceptos, en aras de no ampliar excesivamente las definiciones, dado que, en todo momento, se procederá a realizar la valoración y generación del plan de cuidados por profesionales. Por tanto, la terminología utilizada en el contenido de las guías pretende, únicamente, establecer puntos de referencia para la señalización del camino en la utilización de la aplicación.

\section{CONSIDERACIONES FINALES}

Delante de lo que fue aquí expuesto, puédese considerar que:

$\checkmark \quad$ La atención a la dependencia debe ser abordada de inmediato, haya visto el creciente numero de personas ancianas 
en la población mundial, especialmente aquellas que presenta dependencia en la satisfacción de sus necesidades humanas básicas.

$\checkmark$ Se deben considerar los fracasos en otros países, para no caer en los mismos errores.

$\checkmark$ Se debe instar a la población mayor al mantenimiento de su independencia con promoción de la salud.

$\checkmark$ El recurso más interesante, por economía y eficacia, es el propio domicilio y es ahí hacia donde deben dirigirse los esfuerzos. $\checkmark \quad$ Enfermería debe liderar ese programa, potenciando dos áreas: la enfermería escolar y la enfermera gestora de casos.

Para finalizar entendemos que este trabajo de cierto modo es más uno subsidio que contribuirá para el fortalecimiento de saberes en la area de la enfermería geriátrica y gerontológica , pues despertará el interés de los profesionales de la salud en especial enfermeros, estudiantes e investigadores para la problemática de este grupo de personas que han sido negligenciado en las prácticas sociales.

\section{REFERÊNCIAS}

1. OMS [homepage na internet]. OMS 2012. [acesso em 13 abr 2012]. Disponível em: <www.who.int>

2. IMSERSO [homepage na internet]. Libro Blanco del Envejecimiento Activo. [acesso em 3 maio 2012] Disponível em: <http://www.imserso.es/imserso_01/envejecimiento activo/libro_blanco/index.htm $>$

3. IMSERSO [homepage na internet], Asamblea Mundial sobre el Envejecimiento. Plan de acción Internacional de Viena. Sobre el envejecimiento. [acesso em 15 abril 2012] Disponível em: < http://www.imsersomayores.csic.es/documentos/documentos/asamblea-planviena-01.pdf $>$

4. Comisión Europea. Living Labs for user driven open innovation: an overview of the living labs methodology, activities and achievements. Directorate-General for the Information Society and Media. Unit F4: New Infrastucture Paradigms and Experimental Facilities. European Commission, Information Society and Media; 2009.

5. Espanha. Ley n. ${ }^{\circ}$ 39/2006, de 14 de diciembre. De Promoción de la Autonomía Personal y Atención a las Personas en Situación de Dependencia. [legislação na internet] BOE 15 dez 2006. [acesso em 15 abr 2012]. Disponível em: <http://www.boe.es/buscar/doc.php?id= BOE-A-2006-21990>

6. Abellán AG, Esparza C, Castejon P, et al. Epidemiología de la discapacidad y la dependencia en España. Gac Sanit 2011;25(Supl 2):5-11.

7. Zunzunegui MV. Evolución de la discapacidad y la dependencia. Una mirada internacional. Gac Sanit 2011;25(Supl 2):12-20.

8. Williams J, Lyons B, Rowland D. Unmet long-term care needs of elderly people in the community: a review of the literature. Home Health Care Serv Q 1997;16:93-119.

9. Blogpostgrado [homepage na internet]. Previsiones económicas 2011 del Banco Mundial. [acesso em 5 maio
2012]. Disponível em <http://blogcendocpost.esan.edu. $\mathrm{pe} / \mathrm{p}=551>$

10. WHO [homepage na internet]. Datos de estadísticas sanitarias mundiales 2012. [acesso em 23 abr 2012] Disponível em: <http://apps.who.int/bookorders/anglais/ detart1.jsp? codlan $=3 \&$ codcol $=15 \& \operatorname{codcch}=7672>$

11. Ministerio de Sanidad Política Social e Igualdad. Dirección General de Ordenación Profesional. Informe sobre profesionales de cuidados de enfermería. Oferta - Necesidad 2010-2025.Ministerio de Sanidad Política Social e Igualdad. Dirección General de Ordenación Profesional.

12. Abellán AG, Esparza C, Pérez JD. Evolución y estructura de la población en situación de dependencia. Cuad Rel Laboral 2011;29(1):43-67.

13. Kane R A, Kane RL. Evaluación de las necesidades de los ancianos, Guía practica sobre los instrumentos de medición. Barcelona: SG Editores; 1993.

14. Ministerio de Sanidad y Consumo. Guía para la elaboración del programa del anciano en atención primaria de salud. 5. ed. Madrid: Ministerio de Sanidad y Consumo, Dirección General de Planificación Sanitaria; 1989.

15. Mayans JM. Enfermería en cuidados socio-sanitarios. Madrid: Ediciones DAE. 2005.

16. Martinez-Riera JR. Interrelación profesional y continuidad de los cuidados: necesidad vs dificultad. Cult Cuid 2000;4(7-8):166-71.

17. Morilla Herrera JC, Morales Asencio JM, Cuevas Fernández Gallego M, Berrobianco Cobos E, Delgado Romero A. Utilidad y validez de un instrumento basado en indicadores de la Nursing Outcomes Classification como ayuda al diagnóstico de pacientes crónicos de Atención Primaria con gestión ineficiente de la salud propia. An Sist San Navar 2011;34(1):51-61. 